\title{
Advanced FIB/SEM Characterization of Nuclear Materials in the Irradiated Materials Characterization Lab
}

\author{
Daniel Murray, Fei Teng, Narayan Poudel and Krzysztof Gofryk \\ Idaho National Laboratory, Idaho Falls, Idaho, United States
}

The Irradiated Materials Characterization Laboratory (IMCL) is a user facility located at Idaho National Laboratory's Materials and Fuels Complex. The facilities focus is on safe and efficient microstructure and thermal investigations of irradiated nuclear materials. This is achieved by placing advanced characterization instruments in customizable radiological shielding (Figure 1). Among many other instruments, IMCL currently houses two plasma and one gallium focused ion beam/scanning electron microscope (FIB/SEM). The instruments enable detailed microstructural characterization and site-specific sub-sample removal of irradiated and unirradiated nuclear materials. This has opened the door for the development of new methods to probe the features of these unique and extremely complex materials.

Prospects for advanced next-generation reactors demand a solid fundamental understanding of the physical properties of actinide materials and fuels, including transuranic elements. Despite intensive theoretical and experimental efforts, the effect of $5 f$-electrons on transport properties, and their interplay with micro- and meso-scale structures such as grain boundaries, defects, and/or fission products, are still not well understood. ${ }^{1,2}$ The FIB/SEM instrument enables detailed microstructural characterization (phase identification, grain size/orientation, chemistry, etc.), extraction of single crystal samples, micromanipulation of specimens and other materials, and deposition of conductive materials. Using the FIB/SEM we have developed methods to directly probe site specific electrical and thermal transport properties over a wide temperature and magnetic field ranges. This is accomplished by coupling FIB/SEM methods with the newly developed $3 \omega$-technique. ${ }^{3}$ This in turn, provides transport properties that can be directly correlated with the materials microstructure.

This new experimental setup, shown in Figure 2, required a sapphire microchip support to be fabricated. This is used to couple the FIB/SEM and Physical Properties Measurement System (transport measurements). A selected area of the bulk sample is removed using in-plane lift-out methods and placed onto the microchip support. Then, Platinum deposition is used to deposit electrical contacts in a four-wire configuration. We have developed software to control the measurements and performed several tests on surrogate materials such as $\mathrm{SmB}_{6}$. The obtained thermal conductivity of the micromachined $\mathrm{SmB}_{6}$ agrees well with the literature data. Moreover, its magneto transport properties agree with the presence of the topological insulating ground state in this material. We are currently conducting similar experiments on fuels and other actinide materials. 

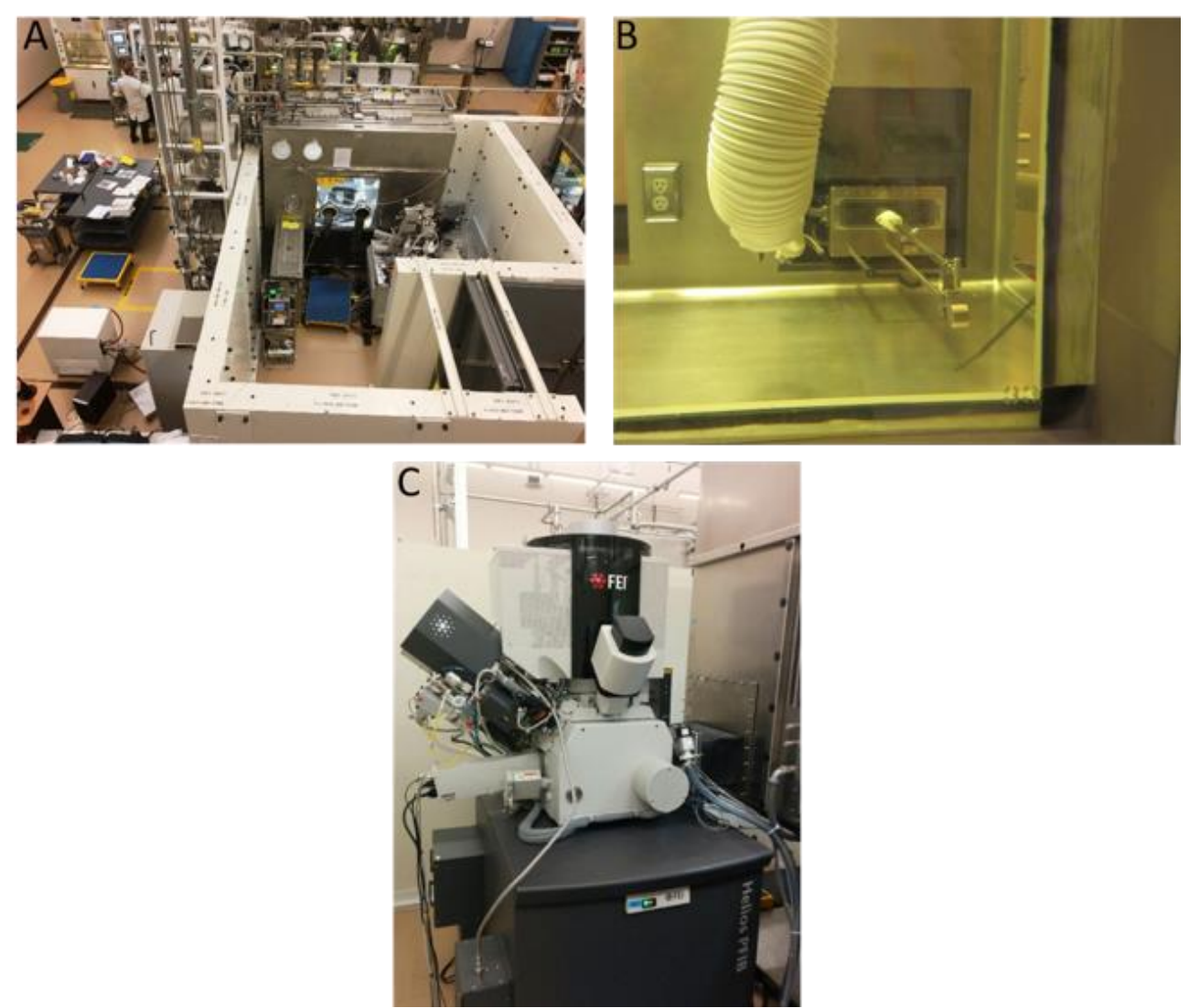

Figure 1. A) Layout of the FIB/SEM instrument shielding in IMCL B) Entry lock used for loading the FIB/SEM as seen through the shielded windows C) Plasma FIB instrument in the shielded cell and coupled to the glove box.
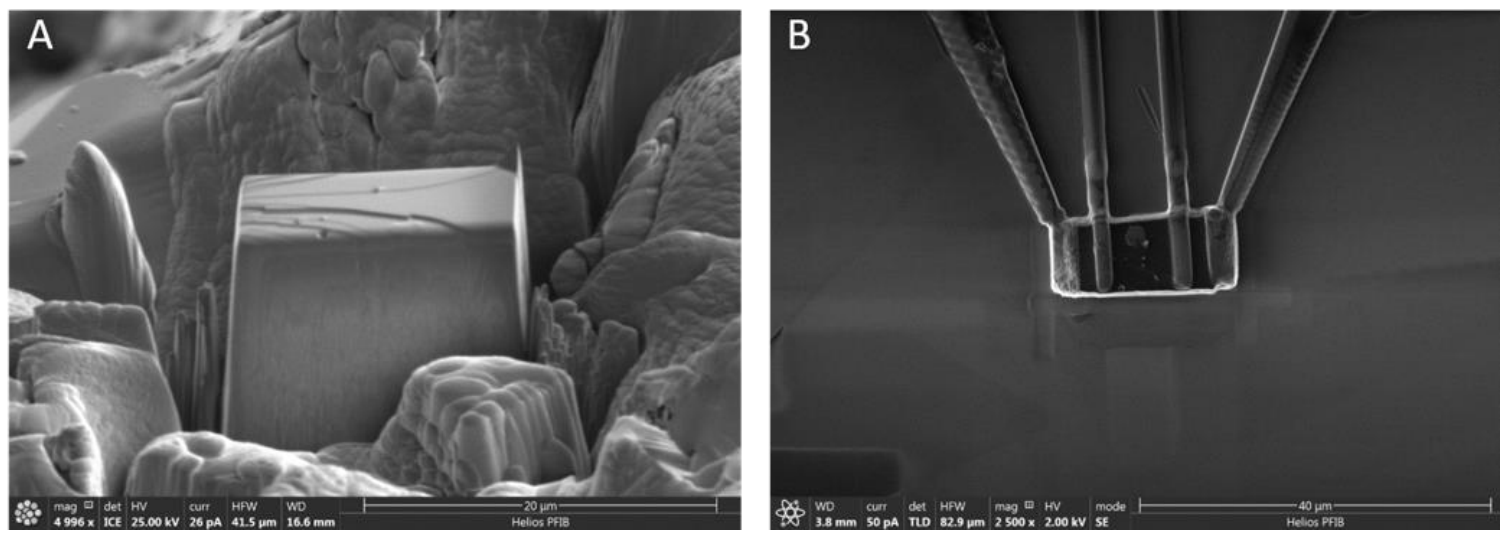

Figure 2. Method for removal of a selected grain within a polycrystalline material. A) A selected grain that has been isolated by ion milling and is ready to be removed from the bulk. B) Finished sample that is ready for electrical and thermal transport testing by AC methods.

\section{References}

1. Moore, K. T., and van der Laan, G., Reviews of Modern Physics (2009) 81 (1), 235

2. Yin, Q., et al., Physical Review B (2011) 84 (19)

3. Shrestha, K., and Gofryk, K., Rev Sci Instrum (2018) 89 (4), 043905 